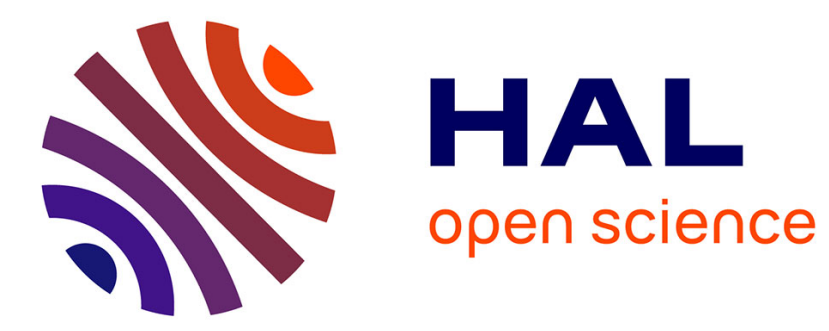

\title{
Modeling of EM Wave Coherent Scattering From a Rough Multilayered Medium With the Scalar Kirchhoff Approximation for GPR Applications
}

Nicolas Pinel, Cédric Le Bastard, Christophe Bourlier

\section{- To cite this version:}

Nicolas Pinel, Cédric Le Bastard, Christophe Bourlier. Modeling of EM Wave Coherent Scattering From a Rough Multilayered Medium With the Scalar Kirchhoff Approximation for GPR Applications. IEEE Transactions on Geoscience and Remote Sensing, 2020, 58 (3), pp.1654-1664. 10.1109/TGRS.2019.2947356 . hal-02370208

\section{HAL Id: hal-02370208 \\ https://hal.science/hal-02370208}

Submitted on 3 Feb 2020

HAL is a multi-disciplinary open access archive for the deposit and dissemination of scientific research documents, whether they are published or not. The documents may come from teaching and research institutions in France or abroad, or from public or private research centers.
L'archive ouverte pluridisciplinaire HAL, est destinée au dépôt et à la diffusion de documents scientifiques de niveau recherche, publiés ou non, émanant des établissements d'enseignement et de recherche français ou étrangers, des laboratoires publics ou privés. 


\title{
Modeling of EM Wave Coherent Scattering From a Rough Multilayered Medium With the Scalar Kirchhoff Approximation for GPR Applications
}

\author{
Nicolas Pinel $^{\circledR}$, Cédric Le Bastard ${ }^{\circledR}$, \\ and Christophe Bourlier ${ }^{\circledR}$
}

\begin{abstract}
This article presents a new asymptotic modeling of electromagnetic (EM) wave coherent scattering from a rough multilayered medium, based on the scalar Kirchhoff-tangent plane approximation. The proposed EM model is developed to simulate a realistic ground-penetrating radar (GPR) signal that considers the interface roughness of the multilayer. It allows us to investigate the influence of the interface roughness on the amplitude of the GPR echoes coming from the multilayered medium. Sounded multilayered medium generally has a low contrast between the successive layers, so that the multiple reflections inside each layer may be neglected; this assumption will be evaluated. The very low computational burden of this EM method is an important advantage as compared with a rigorous numerical method. First, numerical results in the frequency domain are presented to validate the proposed model, by comparison with a reference method based on the Method of Moments (MoM). Then, numerical results in the time domain are presented to analyze the behavior and performance of this new method, and the impact of both the interface roughness and the medium conductivity on the results.
\end{abstract}

Index Terms-Asymptotic diffraction theory, groundpenetrating radar (GPR), multilayered media, nondestructive testing, rough surfaces.

\section{INTRODUCTION}

$\mathbf{G}$ ROUND-penetrating radar (GPR) is a common tool for nondestructive testing of civil engineering materials [1]-[5], environment, and agriculture [6]-[8]. It allows rapid

Manuscript received November 23, 2018; revised May 17, 2019 and September 11, 2019; accepted September 25, 2019. This work was supported by the French National Research Agency (ANR) through the project ACIMP (Improvement of knowledge of complex media composed of cracks and multiparameter inversion). (Corresponding author: Nicolas Pinel.)

N. Pinel is with the Icam School of Engineering-Nantes campus, 44470 Carquefou, France, and also with the UMR CNRS 6164-Polytech Nantes, Institute of Electronics and Telecommunications of Rennes, Université de Nantes, 44306 Nantes, France (e-mail: nicolas.pinel@icam.fr).

C. Le Bastard is with the Project-Team ENDSUM, Centre for Expertise and Engineering on Risks, Environment, Mobility, Urban and Country Planning (Cerema), 49136 Les Ponts de Cé, France, and also with the UMR CNRS 6164-Polytech Nantes, Institute of Electronics and Telecommunications of Rennes, Université de Nantes, 44306 Nantes, France (e-mail: cedric.lebastard@cerema.fr).

C. Bourlier is with the Institut d'Électronique et de Télécommunications de Rennes (IETR) Laboratory, LUNAM Université, Université de Nantes, 44306 Nantes, France (e-mail: christophe.bourlier@univ-nantes.fr).

Color versions of one or more of the figures in this article are available online at http://ieeexplore.ieee.org.

Digital Object Identifier 10.1109/TGRS.2019.2947356 data collection and is widely used to measure or to estimate media parameters (pavements, bare agricultural fields, soils, and so on). In this article, we focus on a medium that is composed of several layers (multilayers), like in [1] and [3]. This multilayered medium can represent either a civil engineering or an agricultural medium, where the dispersion is considered. The vertical structure of this medium can then be deduced from radar profiles, by means of echo detection and amplitude estimation. Echo detection provides the time-delay estimation (TDE) associated with each interface, whereas amplitude estimation is used to retrieve the wave speed (or the permittivity) within each layer.

Conducting rigorous numerical simulations, like with GPRmax [9] or the propagation inside layer expansion (PILE) [10], [11] and GPILE [12] methods, is an efficient way to study and analyze the electromagnetic (EM) wave propagation inside and scattering from a layered medium [13]. GPRmax [9], which is a finite-difference time-domain (FDTD) method, is a common method for GPR numerical simulations. Nevertheless, this method cannot calculate the contribution of each echo coming from the multiple scattering inside the layers. The numerical method PILE [10], developed for 2 interfaces, and its generalization to $n$ interfaces, GPILE [12], have the great advantage to be able to calculate this contribution. They are also able to consider the roughness of the interfaces easily and without any increase in computational burden. Nevertheless, as a numerical method, it requires a significant computing space and time, and the computational burden strongly increases with the number of layers. The use of an appropriate asymptotic model is then of interest to deal with this problem. Thus, in this article, we propose a new asymptotic modeling of EM wave coherent scattering from the rough multilayered medium with a very low computational burden, which is an extension of the previous work that has been led and validated for two interfaces by the PILE method [14]-[17].

This article focuses on the survey of a rough multilayered medium by GPR. Previous articles [1], [3] have already proposed efficient methods to estimate the thicknesses in pavement survey. However, the roughness of the interfaces was not introduced nor discussed. In the GPR literature, some articles deal with the interface roughness [13], [18]-[25]. 
Sai and Ligthart [18] used the phase variations of the GPR signal to profile 2-D rough surfaces. Nevertheless, like Yarovoy et al. [19], who analyzed the scattered field near a rough air-ground interface, only one interface was considered. Yang and Rappaport [13] analyzed the response of a realistic soil by 2-D FDTD for the rough ground surface scattering only, in order to understand and analyze the phenomenon of transmission through the ground surface. For bare agricultural fields, Lambot et al. [20] analyzed the effect of soil surface roughness on the GPR signal and on the inversion of the soil EM parameters. Jonard et al. [24] combined a fullwaveform GPR model with a roughness model to retrieve the surface soil moisture through a signal inversion. Van der Kruk et al. [22], [25] also considered the roughness in the direct modeling approach (by using a 3-D FDTD modeling) to analyze the influence of the interface roughness on the inversion of dispersive GPR pulse propagation in a surface waveguide. Pinel et al. [23] also analyzed the influence of interface roughness, but to estimate the first two time delays coming from an ultrathin asphalt surfacing (UTAS). The aim was then to estimate the first thickness of the pavement. Giannopoulos and Diamanti [21] proposed to use 2-D and 3-D FDTD GPR modelings to investigate the effects of the variation of the subsurface interface roughness on the GPR signals emanating from one target, in which only one interface has been used. In the GPR literature, generally, the fullwaveform forward models are used to consider the roughness in order to analyze the influence of the latter on the results. However, these methods require a high computational burden, especially in the context of a multilayered medium.

In this article, we propose to develop a new asymptotic analytical model of EM wave coherent scattering from a layered medium with random rough interfaces. The proposed method is an extension of previous work that was led and validated for two interfaces only [14]-[17]. Then, the proposed method is applied to a real case and makes it possible to show the importance of considering the interface roughness in the EM wave scattering from a layered medium.

Section II presents the new asymptotic model of EM wave coherent scattering from the rough-layered medium. In Section III, numerical results are presented: first, in the frequency domain, to validate the model with a Method of Moments (MoM)-based numerical method, and second, in the time domain to analyze the behavior and performance of the proposed method. The new method is applied to realistic scenarios in the field of civil engineering, and the results are discussed. Finally, a conclusion is drawn in Section IV.

\section{Formulation of the Problem: ASYMPTOTIC EM MODELING}

\section{A. Context of the EM Modeling}

The aim of this section is to propose a simple mathematical model for describing the EM scattering from a multi-layered medium, by considering the random roughness of the interfaces. Then, for having a simple model, the easiest means is to deal with this complex problem statistically by using the statistical description of the random rough interfaces, which enables us to derive an analytical equation of the average scattered field. Indeed, otherwise, it would be necessary to generate the surfaces and to deal with this problem with a numerical method, which is in general highly time-consuming (and memory-consuming). As a result, some assumptions must be made in order to be able to obtain a simple mathematical formulation.

Following previous work [16], [17], [23], the context of this article is to deal with coherent scattering from random rough surfaces having small to moderate slopes, which corresponds for slightly rough surfaces to moderate-to-large correlation lengths. Indeed, as we are mainly interested in the scattering of the field at and near around normal incidence $\left(\theta_{\text {inc }}=0\right.$ in Fig. 1) and as the surfaces are slightly rough, we will focus our mathematical developments only on coherent scattering. Compared to other methods based on the small perturbation method (see [26] and references therein), the objective is to obtain simple analytical formulations based on the Kirchhofftangent plane approximation (KA).

From previous work [16], which validated our approach based on an extension of the KA to a stack of two random rough surfaces, here, we will further extend this article to a stack of $n$ random rough surfaces (with $n$ an integer such that $n \geq 2$ ). For having simple expressions of the so-called coherent scattered fields, a further approximation of the KA is used: it assumes small surface slopes and works for small angles (relatively to nadir) and is sometimes called scalar KA (SKA) [16] or zeroth-order KA [17].

In addition, it must be highlighted that, contrary to [14], [15], and [17] and following [16], we will consider only one reflection within each layer. Indeed, in the applications of interest here, the contrast between the permittivities of the successive layers is low. This assumption has the great advantage of making the mathematical writing of each echo contribution much simpler. Finally, as the mathematical derivations are long (see [17], [27, Appendixes B and C]), they will not be presented here. Alternatively, an equivalent qualitative physical approach that follows [14] and [15] is elected here. It is pointed out that this qualitative approach was proven to be consistent with a rigorous numerical approach in a recent article [17].

\section{B. Scattering From Multilayers With Flat Interfaces}

We consider the general case of nonnormal incidence $\theta_{\text {inc }} \neq 0$ and $n$ uncorrelated random rough surfaces separating lossy dielectric homogeneous media $\Omega_{k}$ (with $k \in\{2 ; \ldots$; $n+1\}$ ), as shown in Fig. 1. Note that the upper medium $\Omega_{1}$ is lossless. Physically, for short, it may be said that, for thick enough layers, the two random rough interfaces that delimit a given layer may be considered as uncorrelated. Then, the uncorrelated case cannot represent all configurations, in particular very thin layers, like horizontal cracks within pavements or thin oil slick at the sea surface. We evaluate the (average) far-field scattered fields in the specular direction $\theta_{r}=-\theta_{\text {inc }}$, by taking either flat or rough interfaces and by considering only one reflection inside each layer. As used in [16] and [23], we give the expressions of the so-called 


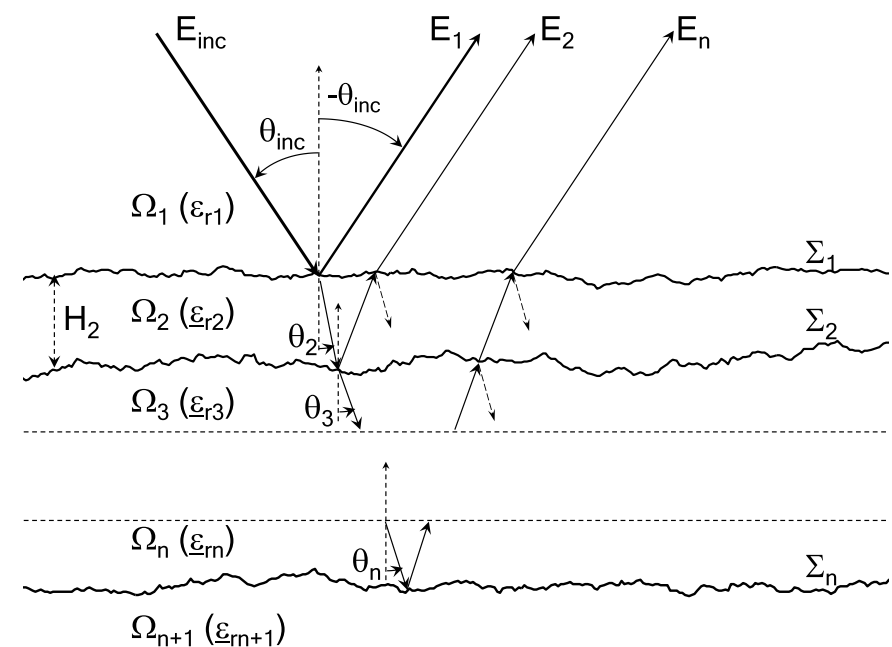

Fig. 1. Illustration of the problem to be solved: coherent scattering from a multi-layered medium made up of $n$ uncorrelated random rough surfaces separating lossy homogeneous media $\Omega_{k}$ (with $k \in\{2 ; \ldots ; n+1\}$ ) (the upper medium $\Omega_{1}$ being lossless), by considering only one reflection inside each layer (i.e., the paths with dashed arrows are not considered).

echoes $s_{k}$ (with $k \in\{1 ; 2 ; \ldots ; n+1\}$ ), which are related to the $k$ th-order scattered field $E_{k}$ and to the incident field $E_{\text {inc }}$ by $s_{k}=E_{k} / E_{\text {inc }} \exp \left(i \phi_{k}\right)$, with $\phi_{k}$ a phase term. First, let us consider the case of flat interfaces. It should be highlighted that one great advantage of calculating the coherent scattering (compared with the incoherent scattering) under the KA is that, for either 1-D or 2-D surfaces, the roughness contribution has the same mathematical expression (see [17], where the developments and validations have been given for 2-D surfaces, compared with [16], where the validations have been given for 1-D surfaces). Then, the expression "roughness contribution" corresponds to the ratio of the coherent scattered field of the rough interface case compared with the flat interface case.

By noting $\theta_{1}=\theta_{\text {inc }}$, the first four echoes are given for flat interfaces as follows:

$$
\begin{aligned}
s_{1}= & r_{12}\left(\theta_{1}\right) e^{i \phi_{1}} \\
s_{2}= & t_{12}\left(\theta_{1}\right) r_{23}\left(\theta_{2}\right) t_{21}\left(\theta_{2}\right) e^{i\left(\phi_{1}+\Delta \phi_{2}\right)} \\
s_{3}= & t_{12}\left(\theta_{1}\right) t_{23}\left(\theta_{2}\right) r_{34}\left(\theta_{3}\right) t_{32}\left(\theta_{3}\right) t_{21}\left(\theta_{2}\right) e^{i\left(\phi_{1}+\Delta \phi_{3}\right)} \\
s_{4}= & t_{12}\left(\theta_{1}\right) t_{23}\left(\theta_{2}\right) t_{34}\left(\theta_{3}\right) r_{45}\left(\theta_{4}\right) t_{43}\left(\theta_{4}\right) t_{32}\left(\theta_{3}\right) t_{21}\left(\theta_{2}\right) \\
& \times e^{i\left(\phi_{1}+\Delta \phi_{4}\right)}
\end{aligned}
$$

with $\Delta \phi_{k}(k \in\{2 ; 3 ; 4\})$ the phase difference of $s_{k}$ with $s_{1}$, $\theta_{\alpha}$ the angle of propagation of the wave inside the medium $\Omega_{\alpha}$, and $t_{\alpha \beta}$ and $r_{\alpha \beta}$ the Fresnel transmission and reflection coefficients from a wave propagating inside the medium $\Omega_{\alpha}$ at the interface with the medium $\Omega_{\beta}$. The angles $\theta_{\alpha}$ are determined by using the well-known transmission Snell-Descartes law. The first phase difference $\Delta \phi_{2}$ is known to be expressed as $\Delta \phi_{2}=2 k_{0} \sqrt{\underline{\epsilon}_{r 2}} \bar{H}_{2} \cos \theta_{2}$, with $k_{0}$ the wavenumber in vacuum and $\bar{H}_{2}$ the thickness of layer $\Omega_{2}$ with flat surfaces $\Sigma_{1}$ and $\Sigma_{2}$. Following [17], it may also be written in a more compact (and more general) form as $\Delta \phi_{2}=2 \kappa_{2 z} \bar{H}_{2}$, with $\kappa_{2 z}=k_{0} \sqrt{\underline{\epsilon}_{r 2}} \cos \theta_{2}$ the vertical component of the propagation vector inside $\Omega_{2}$. It can easily be established that, from an echo $s_{k-1}$ to the following one $s_{k}$, the phase difference has the same form: $2 k_{0} \sqrt{\underline{\epsilon}_{r k}} \bar{H}_{k} \cos \theta_{k}=2 \kappa_{k z} \bar{H}_{k}$, so that the total phase difference of $s_{k}$ with $s_{1}$ takes the form

$$
\Delta \phi_{k}=\sum_{p=2}^{k} 2 k_{0} \sqrt{\underline{\epsilon}_{r p}} \bar{H}_{p} \cos \theta_{p}=\sum_{p=2}^{k} 2 \kappa_{p z} \bar{H}_{p} .
$$

Then, the expression of the scattered echoes $s_{k}$ can easily be generalized to any order $k \in\{2 ; \ldots ; n\}$ as

$$
\begin{aligned}
s_{k}= & {\left[\prod_{p=1}^{k-1} t_{p(p+1)}\left(\theta_{p}\right) t_{(p+1) p}\left(\theta_{p+1}\right)\right] r_{k(k+1)}\left(\theta_{k}\right) e^{i\left(\phi_{1}+\Delta \phi_{k}\right)} } \\
= & {\left[\prod_{p=1}^{k-1} t_{p(p+1)}\left(\theta_{p}\right) t_{(p+1) p}\left(\theta_{p+1}\right) e^{i 2 \kappa_{(p+1) z} \bar{H}_{p+1}}\right] } \\
& \times r_{k(k+1)}\left(\theta_{k}\right) e^{i \phi_{1}} .
\end{aligned}
$$

In what follows, the expressions are generalized to deal with uncorrelated random rough interfaces, by using the SKA.

\section{Coherent Scattering}

Let us now focus on the case of uncorrelated random rough interfaces and study the coherent scattering from such a structure. Following previous mathematical developments based on the KA and reduced to either the method of stationary phase (MSP) [27] or the SKA [17], it can be shown that, for uncorrelated random rough interfaces, the angle of propagation inside a given medium $\Omega_{k}$ is the same as that for perfectly flat surfaces for deriving the coherent scattering in the specular direction $-\theta_{\text {inc }}$ (see Fig. 1). Moreover, in the evaluation of the statistical average $\left\langle s_{k}\right\rangle$ associated with the coherent scattered field $\left\langle E_{k}\right\rangle$, the remaining random variables are the film thicknesses $H_{p}$ (with $p \in\{1 \ldots k\}$ ), which are due to the heights variations of the points of transmission or reflection at the corresponding interfaces $\Sigma_{p-1}$ and $\Sigma_{p}$. We recall here that this approach, which could be described as qualitative, is physically sound and is based on rigorous mathematical developments that have been proven to be valid [17], [27].

Then, evaluating $\left\langle s_{k}\right\rangle$ consists in determining $\left\langle e^{i \Delta \phi_{k}}\right\rangle$, in which the phase term $\Delta \phi_{k}$ can be expressed as $\Delta \phi_{k}=$ $\overline{\Delta \phi_{k}}+\delta \phi_{k}$, with $\overline{\Delta \phi_{k}}$ its statistical average and $\delta \phi_{k}$ its variations around this average. Then, we have $\left\langle e^{i \Delta \phi_{k}}\right\rangle=$ $e^{i \overline{\Delta \phi_{k}}}\left\langle e^{i \delta \phi_{k}}\right\rangle$. Note that the statistical average $\overline{\Delta \phi_{k}}$ is given by (5), with $\bar{H}_{p}$ the mean layer thickness of the layer $\Omega_{p}$. Thus, the difficulty consists in evaluating the statistical average $\left\langle e^{i \delta \phi_{k}}\right\rangle$, which implies to express the phase variations $\delta \phi_{k}$, with $k \in\{2 ; \ldots ; n\}$.

First, recall that for the first echo $s_{1}$, as $\Sigma_{1}$ is a random rough surface, the phase term $\phi_{1}$ also becomes a random variable, whose variations $\delta \phi_{1}$ are given by [15, eq. (14)]

$$
\delta \phi_{1}=2 \kappa_{1 z} \delta \zeta_{1}
$$

For $k=2$, the expression of $\delta \phi_{2}$ has been established in previous work [15, eq. (19)]

$$
\begin{aligned}
\delta \phi_{2}=k_{0}\left(\delta \zeta_{1}+\delta \zeta_{1^{\prime}}\right)\left(\sqrt{\epsilon_{r 1}} \cos \theta_{1}-\sqrt{\underline{\epsilon}_{r 2}} \cos \theta_{2}\right) \\
+2 k_{0} \sqrt{\underline{\epsilon}_{r 2}} \delta \zeta_{2} \cos \theta_{2}
\end{aligned}
$$


with $\delta \zeta_{1}$ the height variations of the surface $\Sigma_{1}$ around its mean plane for the wave entering down into $\Omega_{2}, \delta \zeta_{2}$ the ones of the surface $\Sigma_{2}$, and $\delta \zeta_{1^{\prime}}$ the ones of the surface $\Sigma_{1}$ around its mean plane for the wave going back into $\Omega_{1}$. It can be rewritten in a more compact form as

$$
\delta \phi_{2}=\left(\kappa_{1 z}-\kappa_{2 z}\right)\left(\delta \zeta_{1}+\delta \zeta_{1^{\prime}}\right)+2 \kappa_{2 z} \delta \zeta_{2}
$$

Based on this previous work, this formulation can be extended to $\delta \phi_{3}$ as

$$
\begin{array}{r}
\delta \phi_{3}=\left(\kappa_{1 z}-\kappa_{2 z}\right)\left(\delta \zeta_{1}+\delta \zeta_{1^{\prime}}\right)+\left(\kappa_{2 z}-\kappa_{3 z}\right)\left(\delta \zeta_{2}+\delta \zeta_{2^{\prime}}\right) \\
+2 \kappa_{3 z} \delta \zeta_{3} .
\end{array}
$$

Then, it can be generalized to $\delta \phi_{k}$ with $k \in\{2 ; \ldots ; n\}$ as

$$
\delta \phi_{k}=2 \kappa_{k z} \delta \zeta_{k}+\sum_{p=1}^{k-1}\left(\kappa_{p z}-\kappa_{(p+1) z}\right)\left(\delta \zeta_{p}+\delta \zeta_{p^{\prime}}\right) .
$$

The statistical evaluation of these phase terms is then derived in what follows.

\section{Expressions Under the SKA for Gaussian Statistics}

By assuming Gaussian statistics, the statistical average over the first echo $s_{1}$ reduces to the statistical average over $\delta \phi_{1}$ as [15], [17]

$$
\left\langle e^{i \delta \phi_{1}}\right\rangle=\exp \left(-2 \kappa_{1 z}^{2} \sigma_{h 1}^{2}\right)=\exp \left(-2 R a_{r 12}^{2}\right)
$$

with $\sigma_{h 1}$ the rms height of the upper surface $\Sigma_{1}$, in which the Rayleigh roughness parameter can be defined as $R a_{r 12}=$ $\kappa_{1 z} \sigma_{h 1}$ for the reflection inside the medium $\Omega_{1}$ with an angle $\theta_{1}$ onto the surface $\Sigma_{1}$ separating the medium $\Omega_{2}$.

For the second echo $s_{2}$, as the surface points are assumed to be uncorrelated, $\delta \zeta_{1}, \delta \zeta_{1^{\prime}}$, and $\delta \zeta_{2}$ are independent random variables. As a consequence, the evaluation of $\left\langle e^{i \delta \phi_{1}}\right\rangle$ becomes simple. Indeed

$$
\left\langle e^{i \delta \phi_{2}}\right\rangle=\left\langle e^{i\left(\kappa_{1 z}-\kappa_{2 z}\right) \delta \zeta_{1}}\right\rangle\left\langle e^{i\left(\kappa_{1 z}-\kappa_{2 z}\right) \delta \zeta_{1^{\prime}}}\right\rangle\left\langle e^{i 2 \kappa_{2 z} \delta \zeta_{2}}\right\rangle .
$$

Then, this evaluation can be reduced to [15], [17]

$$
\begin{aligned}
\left\langle e^{i \delta \phi_{2}}\right\rangle & =e^{-\left(\kappa_{1 z}-\kappa_{2 z}\right)^{2} \sigma_{h 1}^{2}} e^{-2 \kappa_{2 z}^{2} \sigma_{h 2}^{2}} \\
& =e^{-4 R a_{t 12^{2}}} e^{-2 R a_{r 23}{ }^{2}}
\end{aligned}
$$

in which $R a_{r 23}=\kappa_{2 z} \sigma_{h 2}$ is also a Rayleigh roughness parameter in reflection, but for the reflection inside the medium $\Omega_{2}$ with an angle $\theta_{2}$ onto the surface $\Sigma_{2}$ separating the medium $\Omega_{3}$. Moreover, the Rayleigh roughness parameter in transmission, for the transmission from the medium $\Omega_{1}$ with an angle $\theta_{1}$ through the surface $\Sigma_{1}$ into the medium $\Omega_{2}$, is introduced: it is then given by $R a_{t 12}=\frac{\sqrt{\left(\kappa_{1 z}-\kappa_{2 z}\right)^{2}}}{2} \sigma_{h 1}$ [15], [27].

Then, for Gaussian statistics and under the same assumption of uncorrelated surface points, the extension to the third echo $s_{3}$ is made easy. It is given by

$$
\begin{aligned}
\left\langle e^{i \delta \phi_{3}}\right\rangle & =e^{-\left(\kappa_{1 z}-\kappa_{2 z}\right)^{2} \sigma_{h 1}^{2}} e^{-\left(\kappa_{2 z}-\kappa_{3 z}\right)^{2} \sigma_{h 2}^{2}} e^{-2 \kappa_{3 z}^{2} \sigma_{h 3}^{2}} \\
& =e^{-4 R a_{t 12}{ }^{2}} e^{-4 R a_{t 23}{ }^{2}} e^{-2 R a_{r 34}{ }^{2}}
\end{aligned}
$$

with $R a_{t 23}=\frac{\sqrt{\left(\kappa_{2 z}-\kappa_{3 z}\right)^{2}}}{2} \sigma_{h 2}$ for the transmission from $\Omega_{2}$ into $\Omega_{3}$ and $R a_{r 34}=\kappa_{3 z} \sigma_{h 3}$ for the reflection inside $\Omega_{3}$ and onto $\Sigma_{3}$.
Thus, by keeping the same assumption of uncorrelated random rough interfaces, the expression can be generalized for any echo $s_{k}$ (with $k \in\{2 ; \ldots ; n\}$ ) as

$$
\left\langle e^{i \delta \phi_{k}}\right\rangle=e^{-2 R a_{r k(k+1)}{ }^{2}} \prod_{p=1}^{k-1} e^{-4 R a_{t p(p+1)^{2}}}
$$

with $R a_{t p(p+1)}=\frac{\sqrt{\left(\kappa_{p z}-\kappa_{(p+1) z}\right)^{2}}}{2} \sigma_{h p}$ for the transmission from $\Omega_{p}$ into $\Omega_{p+1}$ through the surface $\Sigma_{p}$ and $R a_{r k(k+1)}=$ $\kappa_{k z} \sigma_{h k}$ for the reflection inside $\Omega_{k}$ and onto $\Sigma_{k}$ separating the medium $\Omega_{k+1}$. Accordingly, a global Rayleigh roughness parameter $R a_{k}$ associated with any echo $s_{k}$ can be defined with respect to the elementary reflection and transmission Rayleigh roughness parameters for Gaussian statistics such that $\left\langle e^{i \delta \phi_{k}}\right\rangle=e^{-2 R a_{k}^{2}}$. It is given by

$$
R a_{k}^{2}=R a_{r k(k+1)}{ }^{2}+\sum_{p=1}^{k-1} 2 R a_{t p(p+1)}{ }^{2} \quad \forall k \in\{2 ; \ldots ; n\} .
$$

Note that the case $k=2$ reduces to the second echo in [16] [see (5)]. Thus, under such conditions (in particular, for uncorrelated roughness of the interfaces), the great advantage of the SKA method is that it makes it possible to obtain an analytical expression of each echo $s_{k}$. It is also noticeable that the Rayleigh roughness parameters are independent of the autocorrelation of the rough interfaces. This feature is a valid approximation for small-to-moderate surface slopes.

In Section III, numerical results are presented to analyze the influence of the interface roughness on the frequency and time domain scattered fields. They begin with a validation of this extended method for the case of three random rough interfaces, by comparison with the GPILE method [12]. The hypothesis of neglecting the multiple reflections inside each layer is also evaluated.

\section{NUMERICAL ReSUlts AND Discussion}

To analyze the proposed method, simulations are carried out in the field of civil engineering in the frequency and time domains. The simulation parameters are chosen to match a conventional GPR configuration, an air-coupled radar configuration at vertical incidence (nadir, $\theta_{\mathrm{inc}}=0$ in Fig. 1).

\section{A. Validation by Comparison With GPILE Method}

First, numerical comparisons with a rigorous numerical method based on the MoM are conducted in order to assess the validity of the proposed analytical SKA approach. For doing so, we need to dispose of a numerical reference method that is able to distinguish the contribution of each reflection inside the multilayered medium. In this context, available methods that match these constraints are not numerous. Here, we will consider the recently published GPILE method [12]. This method is an extension of the PILE method [10], [28], which deals with the scattering from two interfaces, to the case of three (and even $n \geq 3$ ) interfaces. Then, the following validation is restricted to a configuration of three interfaces. 

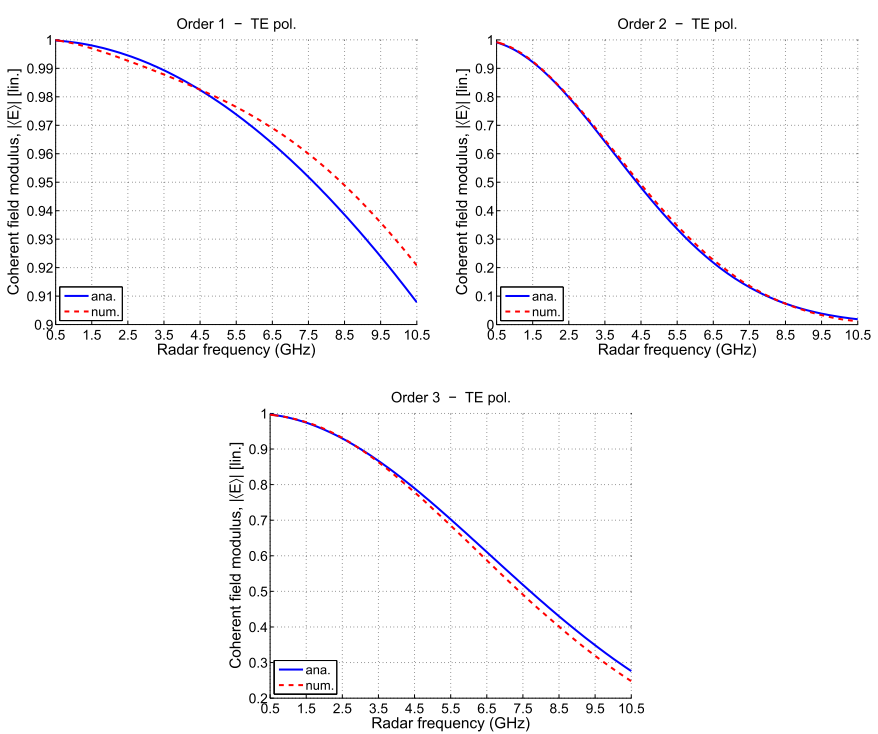

Fig. 2. Modulus of the coherent fields scattered from a multilayered medium of three random rough interfaces, in the frequency band $f \in[0.5 ; 10.5] \mathrm{GHz}$ : comparison of the proposed analytical model with a reference numerical method.

The simulation parameters are as follows: the upper medium is the air, which is assimilated to the vacuum (relative permittivity $\epsilon_{r 1}^{\prime}=1$, conductivity $\sigma_{1}=0$ ). The two inner layers of thicknesses $\bar{H}_{2}=40 \mathrm{~mm}$ and $\bar{H}_{3}=20 \mathrm{~mm}$ are characterized by relative permittivities $\epsilon_{r 2}^{\prime}=4.5$ and $\epsilon_{r 3}^{\prime}=3$ and conductivities $\sigma_{2}=10^{-3} \mathrm{~S} / \mathrm{m}$ and $\sigma_{3}=10^{-2} \mathrm{~S} / \mathrm{m}$, respectively. The lower medium has a relative permittivity $\epsilon_{r 4}^{\prime}=7$ and conductivity $\sigma_{4}=5 \times 10^{-3} \mathrm{~S} / \mathrm{m}$. The three uncorrelated random rough interfaces are assumed to obey a Gaussian process with rms heights $\sigma_{h 1}=1 \mathrm{~mm}, \sigma_{h 2}=3 \mathrm{~mm}$, and $\sigma_{h 3}=2 \mathrm{~mm}$, and correlation lengths $L_{c 1}=10 \mathrm{~mm}, L_{c 2}=$ $30 \mathrm{~mm}$, and $L_{c 3}=30 \mathrm{~mm}$. Then, numerical simulations of the different contributions of the backscattered field from this multilayered structure are conducted at normal incidence in the frequency domain, for a large range of frequencies: $f \in[0.5 ; 10.5] \mathrm{GHz}$ (with $0.01-\mathrm{GHz}$ frequency step). The polarization is horizontal (or transverse electric, TE). For the numerical GPILE method, 50 independent surfaces realizations of length $L=5 \mathrm{~m}$ are used in order to compute the coherent backscattered fields, and the Thorsos tapered beam is applied, with main parameter $g=L / 6$, in order to make the contribution of the edges of the interfaces negligible (for more details, see [23]). It may be noted that the configuration is very similar to that in [11] for the two upper layers, where the multiple scattering effect has been shown to be negligible.

The associated results are shown in Fig. 2, in which the first subfigure corresponds to the contribution of the roughness to $s_{1}$ (modulus-upper interface only), the second to that of $s_{2}$ (modulus-first two interfaces only), and the third to that of $s_{3}$ (modulus). As stipulated before (1), this means that the results plot the ratio of the coherent scattered field of the rough interface case compared with the flat interface case. Then, the first two figures in Fig. 2 correspond to cases that have already been validated [16], contrary to the third one. It can be seen that, similar to orders 1 and 2 , order 3 shows very
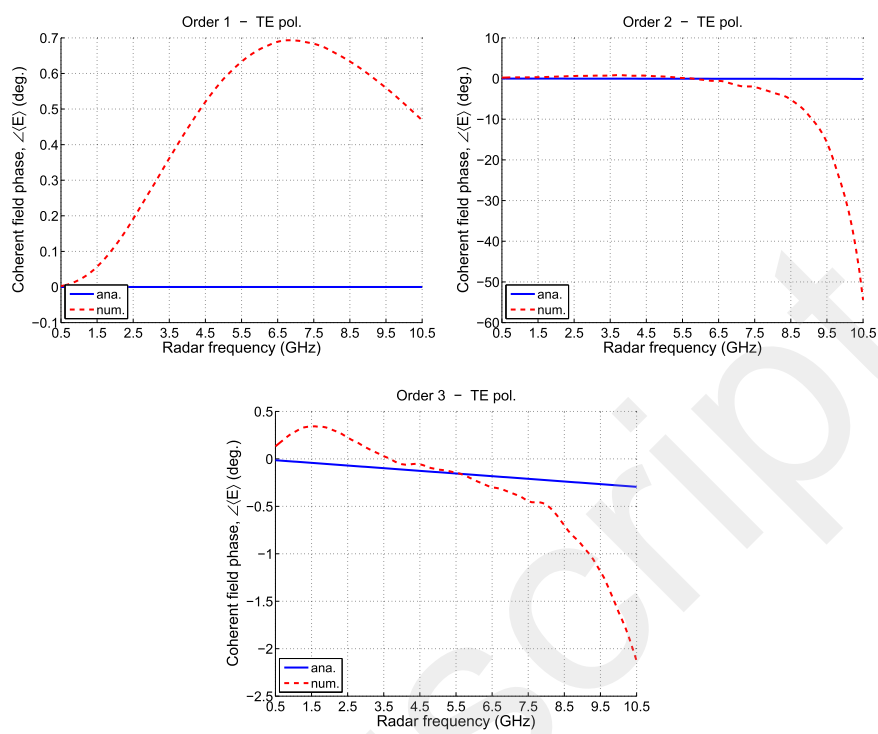

Fig. 3. Phase (in degrees) of the coherent fields scattered from a multilayered medium of three random rough interfaces, in the frequency band $f \in$ $[0.5 ; 10.5] \mathrm{GHz}$ : comparison of the proposed analytical model with a reference numerical method.
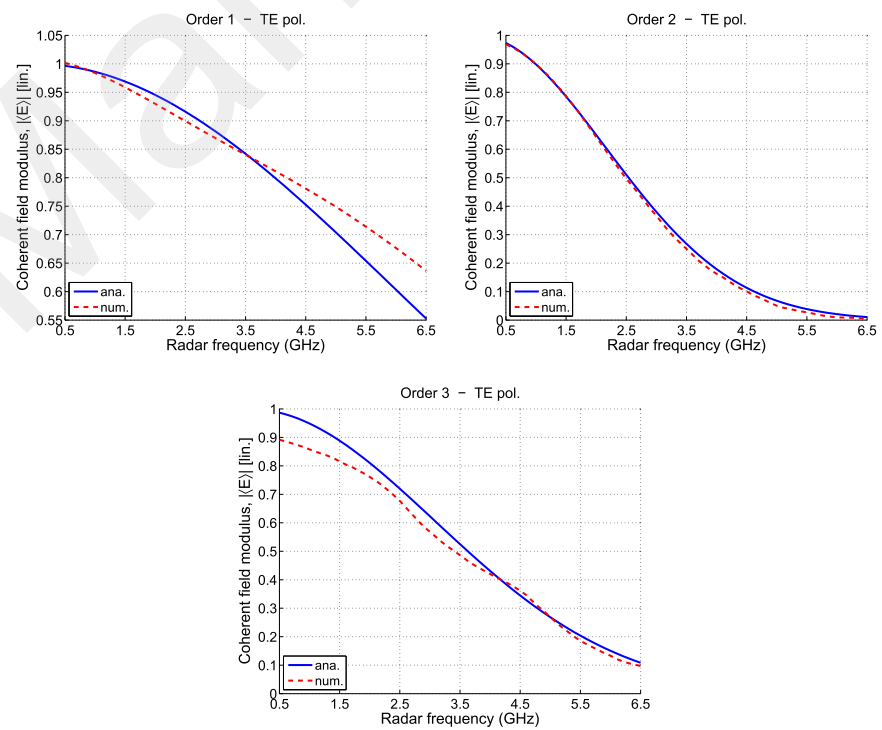

Fig. 4. Modulus of the coherent fields scattered from a multilayered medium of three random rough interfaces, in the frequency band $f \in$ [0.5; 6.5] GHz: the same parameters as in Fig. 2, except for the interface roughness: $\sigma_{h 1}=4 \mathrm{~mm}, \sigma_{h 2}=5 \mathrm{~mm}$, and $\sigma_{h 3}=4 \mathrm{~mm}$.

good performances of the proposed analytical model compared with the reference numerical method. Only slight differences appear between the two methods for the higher frequencies. The same qualitative analysis and conclusions can be made for the phase in Fig. 3. The only exception concerns the higher frequencies of the second order, but it is not of importance as the level of its modulus reaches negligible levels. In addition, this behavior was expected, as the SKA is a low-frequency asymptotic model.

Simulations for larger $\mathrm{rms}$ heights $\left(\sigma_{h 1}=4 \mathrm{~mm}\right.$, $\sigma_{h 2}=5 \mathrm{~mm}$, and $\sigma_{h 3}=4 \mathrm{~mm}$ ) and the same correlation lengths are shown in Fig. 4 for the modulus and in Fig. 5 

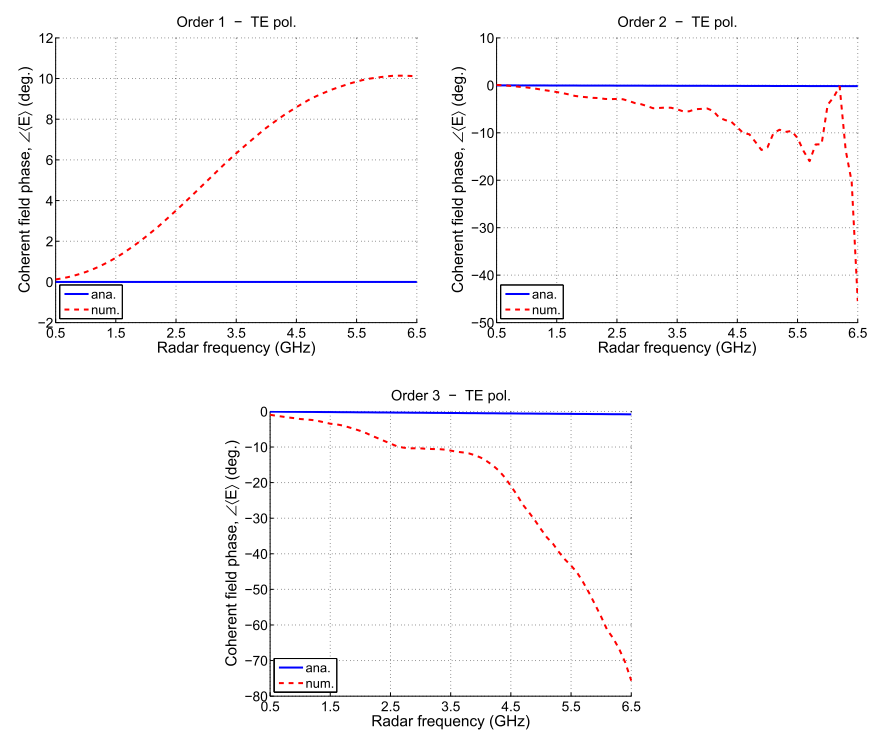

Fig. 5. Phase of the coherent fields scattered from a multilayered medium of three random rough interfaces, in the frequency band $f \in[0.5 ; 6.5] \mathrm{GHz}$ : the same parameters as in Fig. 3, except for the interface roughness: $\sigma_{h 1}=4 \mathrm{~mm}$, $\sigma_{h 2}=5 \mathrm{~mm}$, and $\sigma_{h 3}=4 \mathrm{~mm}$.

for the phase. Compared with the first simulation, we will restrict the frequency range to $f \in[0.5 ; 6.5] \mathrm{GHz}$ (with $0.1-\mathrm{GHz}$ frequency step), as the time consumption increases with the frequency. They show an overall good agreement with the reference numerical method, despite the fact that the assumption of small slopes is not fully valid anymore. The main degradation concerns the phase, but it does not strongly affect the total field.

For the third simulation, we study the influence of the multiple reflections inside the two inner layers, by computing the GPILE method for a number of inner reflections up to 4 . Physically and following [11], it is reasonable to consider it as enough for taking all contributing inner multiple reflections into account. We changed the following physical/dielectric parameters for (and keep the other ones constant): $\bar{H}_{3}=$ $150 \mathrm{~mm}, \epsilon_{r 2}^{\prime}=4, \epsilon_{r 3}^{\prime}=5, \epsilon_{r 4}^{\prime}=4, \sigma_{2}=5 \times 10^{-3} \mathrm{~S} / \mathrm{m}$, $\sigma_{4}=10^{-2} \mathrm{~S} / \mathrm{m}, \sigma_{h 2}=2 \mathrm{~mm}$, and $\sigma_{h 3}=2.3 \mathrm{~mm}$.

The associated results are shown in Fig. 6 for the modulus and in Fig. 7 for the phase. In each figure, the fourth subfigure (bottom-right subfigure) shows the cumulative contributions of all orders $1-3$, and they are compared with the "total" contribution $\left(p_{\text {PILE }}=4\right)$. Similar to the first case, the results of each contribution are in very good agreement with the reference numerical method, and in particular for the newly calculated third contribution. In addition, the comparison of orders 1-3 with the "total" contribution shows only slight differences, even when computing the phase. In fact, there is no observable difference between the numerical methods, which computes only the primary echoes and that which computes all the echoes.

In order to study the influence of the incidence angle, Figs. 8 and 9 show the numerical results for the same parameters as in Figs. 2 and 3, but for a fixed frequency $f=5 \mathrm{GHz}$, and by varying the incidence angle from normal incidence
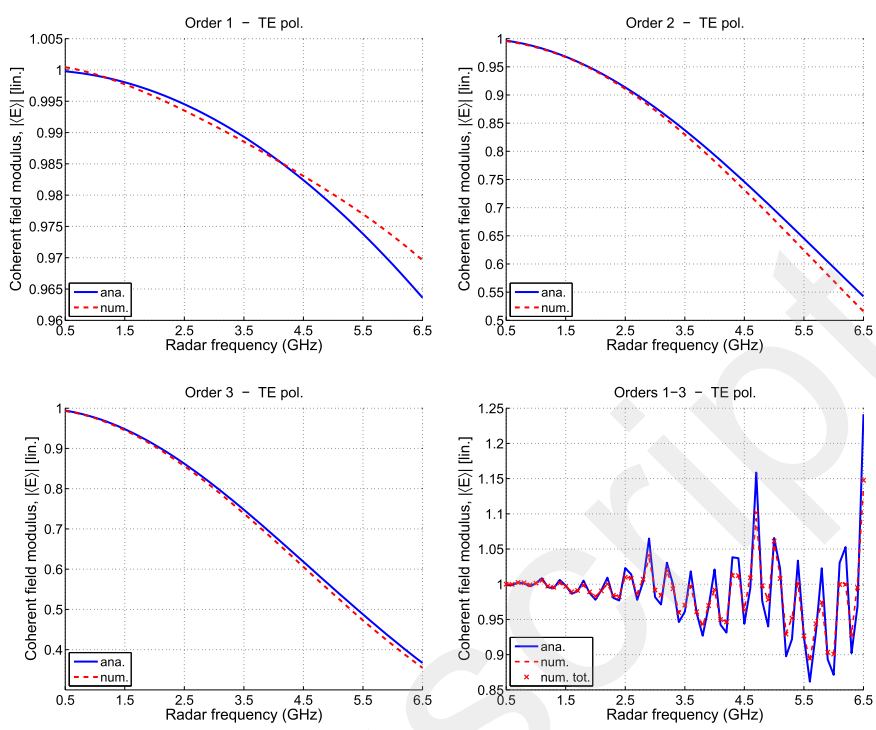

Fig. 6. Modulus of the coherent fields scattered from a multilayered medium of three random rough interfaces under the third configuration, in the frequency band $f \in[0.5 ; 6.5] \mathrm{GHz}$ : comparison of the proposed analytical model with a reference numerical method.
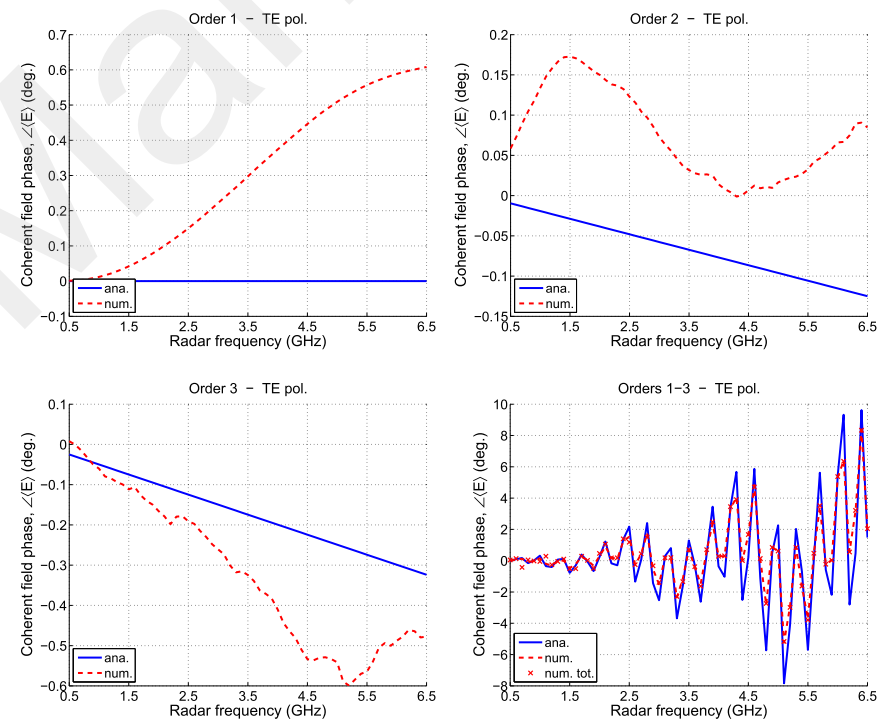

Fig. 7. Phase (in degrees) of the coherent fields scattered from a multilayered medium of three random rough interfaces under the third configuration, in the frequency band $f \in[0.5 ; 6.5] \mathrm{GHz}$ : comparison of the proposed analytical model with a reference numerical method.

(like in Figs. 2 and 3) down to $80^{\circ}$. The TM polarization is also shown in Figs. 10 and 11.

It can be seen that the model correctly captures the general behavior of the modulus of each order contribution in the whole angular range (which is wide), except for the first order in TM pol. around Brewster incidence (recall that order 1 corresponds to the classical case of the scattering from a single interface). About the phase, like for frequency domain results, the behavior is not correctly captured, but the values are only of a few degrees, so that it does not affect much the general agreement (again, except for TM pol. and order 1 around Brewster incidence). Thus, except for the Brewster 

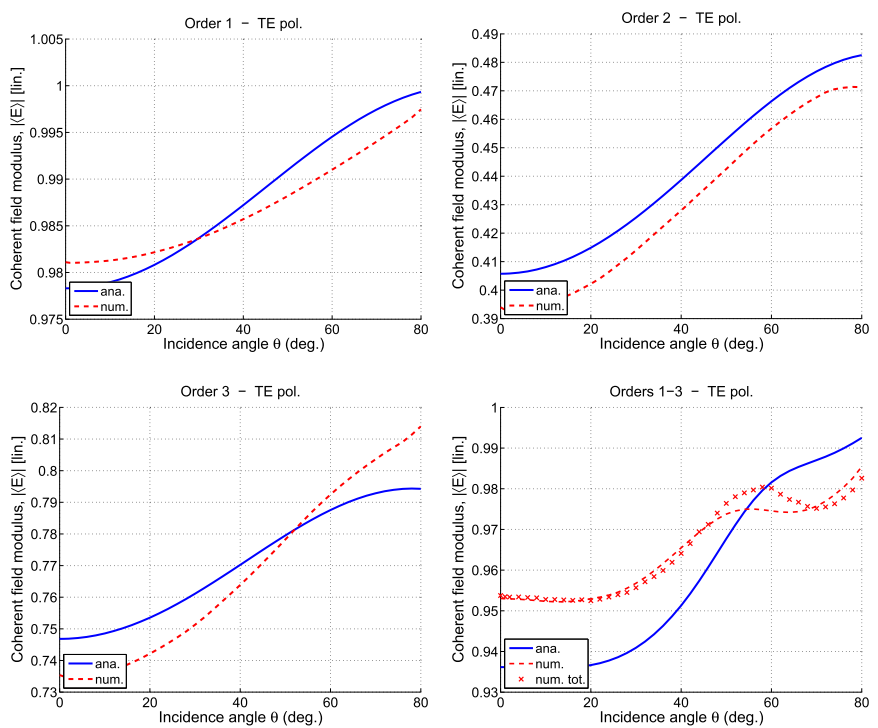

Fig. 8. Modulus of the coherent fields scattered from a multilayered medium of three random rough interfaces versus the incidence angle, for a frequency $f=5 \mathrm{GHz}$ (the same other parameters as in Fig. 2).
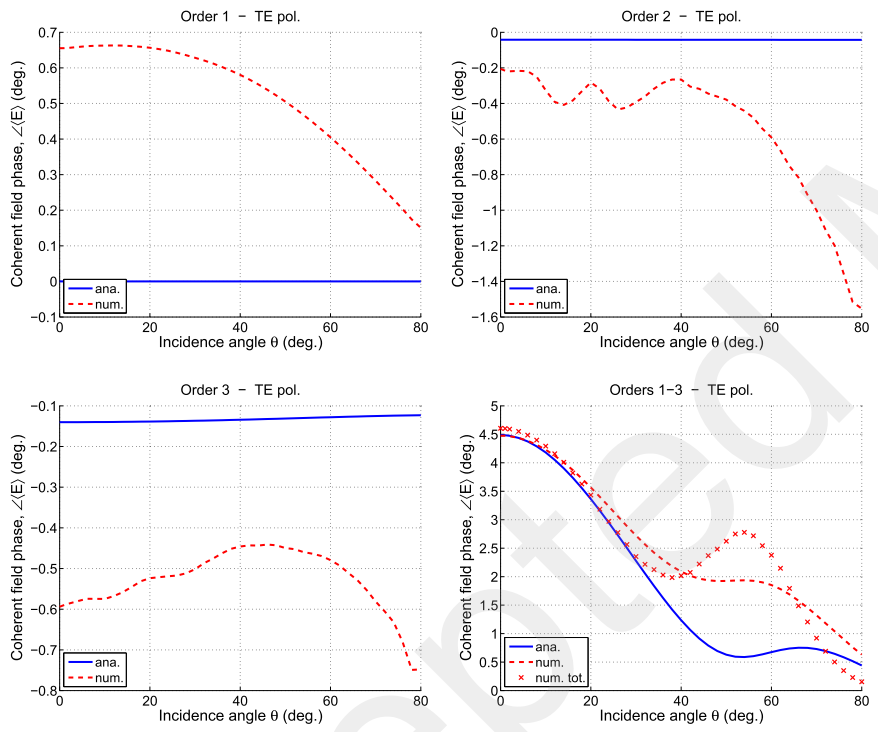

Fig. 9. Phase of the coherent fields scattered from a multilayered medium of three random rough interfaces versus the incidence angle, for a frequency $f=5 \mathrm{GHz}$ (the same other parameters as in Fig. 3).

phenomenon and for order 1 , the model is not so much restricted in terms of angular validity domain, contrary to what its name suggests. Note that for low-grazing angles $\left(\theta_{i} \rightarrow 90^{\circ}\right)$, the model is not valid anymore, because the shadowing and multiple scattering effects are not considered.

Thus, this validates the proposed extension of the SKA to at least three interfaces, together with the assumption of taking only single reflections inside each layer. In Section III-B, additional numerical results are given for more layers, in both the frequency and time domains. The results will be presented only for the SKA, as it would be too much time- and spaceconsuming for the numerical reference method.
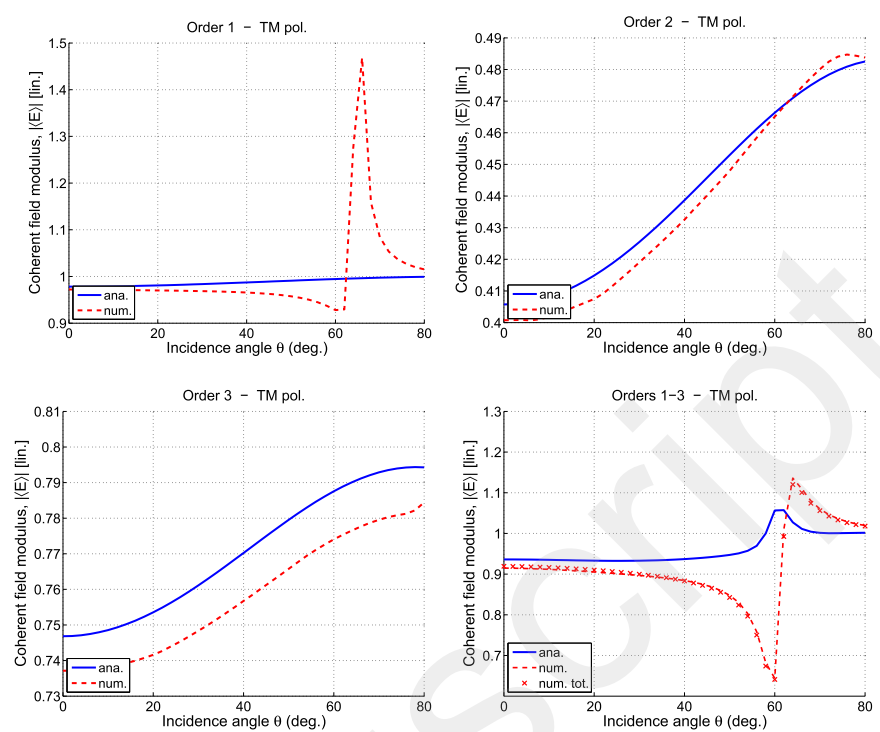

Fig. 10. Results of the modulus for the same parameters as in Fig. 8, but for TM polarization.
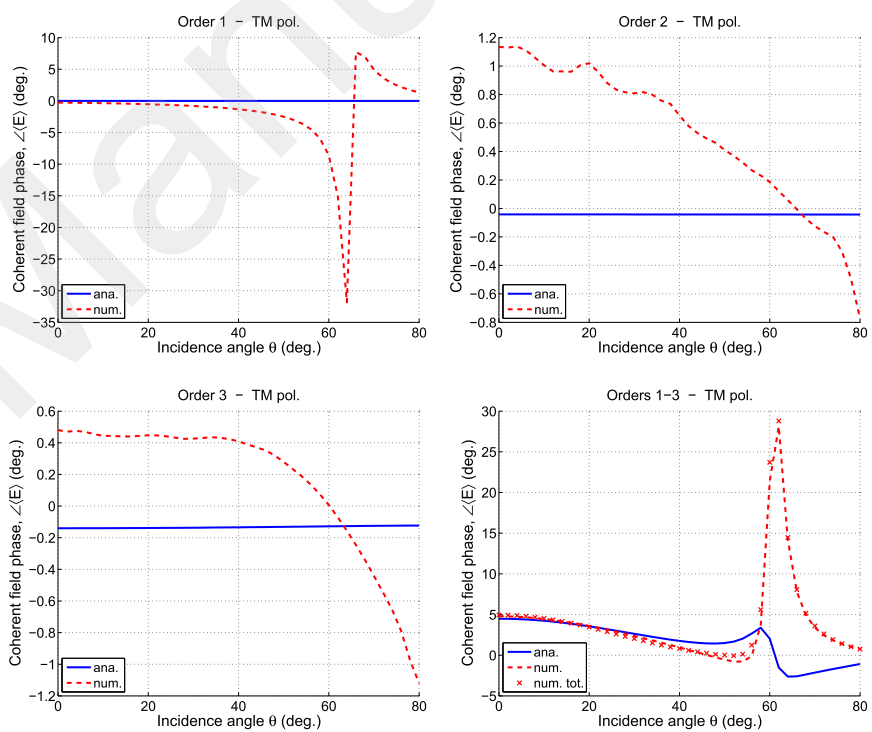

Fig. 11. Results of the phase for the same parameters as in Fig. 9, but for TM polarization.

TABLE I

Parameters of the Multilayered Medium: For Each Medium $\Omega_{k}$ (See Fig. 1), Mean Thickness $\bar{H}$, Real Relative Permittivity $\epsilon_{r}^{\prime}$, CONDUCTIVITY $\sigma$, AND SURFACE rms Height $\sigma_{h}$ ARE AsSOCIATEd With INTERFACE $\Sigma_{k}$

\begin{tabular}{|c|c|c|c|c|} 
Medium \# & $\bar{H}(\mathrm{~mm})$ & $\epsilon_{r}^{\prime}$ & $\sigma(\mathrm{S} / \mathrm{m})$ & $\sigma_{h}(\mathrm{~mm})$ \\
\hline 1 & $\infty$ & 1 & 0 & 1.0 \\
\hline 2 & 40 & 4 & $5 \times 10^{-3}$ & 2.0 \\
\hline 3 & 150 & 5 & $1 \times 10^{-2}$ & 2.3 \\
\hline 4 & 75 & 4 & $1 \times 10^{-2}$ & 4.0 \\
\hline 5 & 150 & 9 & $1 \times 10^{-2}$ & 4.0 \\
\hline 6 & $\infty$ & 11 & $1 \times 10^{-2}$ & -
\end{tabular}

\section{B. Numerical Results for More Layers}

The studied medium structure is made up of five layers like in [3]. The used values of thickness, permittivity, conductivity, and roughness standard deviation are reported in Table I. Different frequency bands are studied: [0.5; 2.5], [0.5; 4.5], 


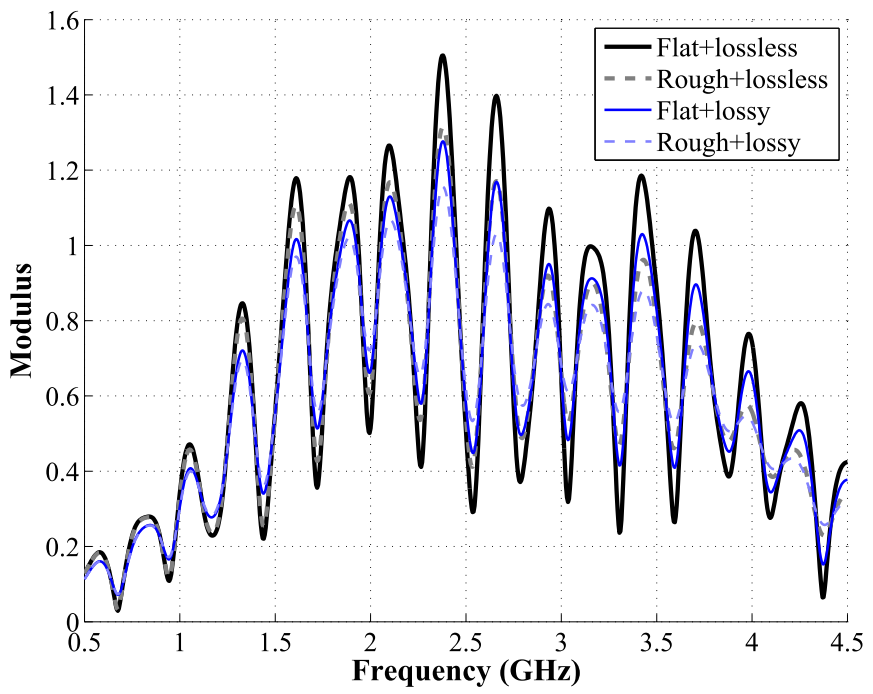

Fig. 12. Modulus (in $\mathrm{V} / \mathrm{m}$ ) of the GPR signal in the frequency domain for the four cases (1-4), in the frequency band $f \in[0.5 ; 4.5] \mathrm{GHz}$.

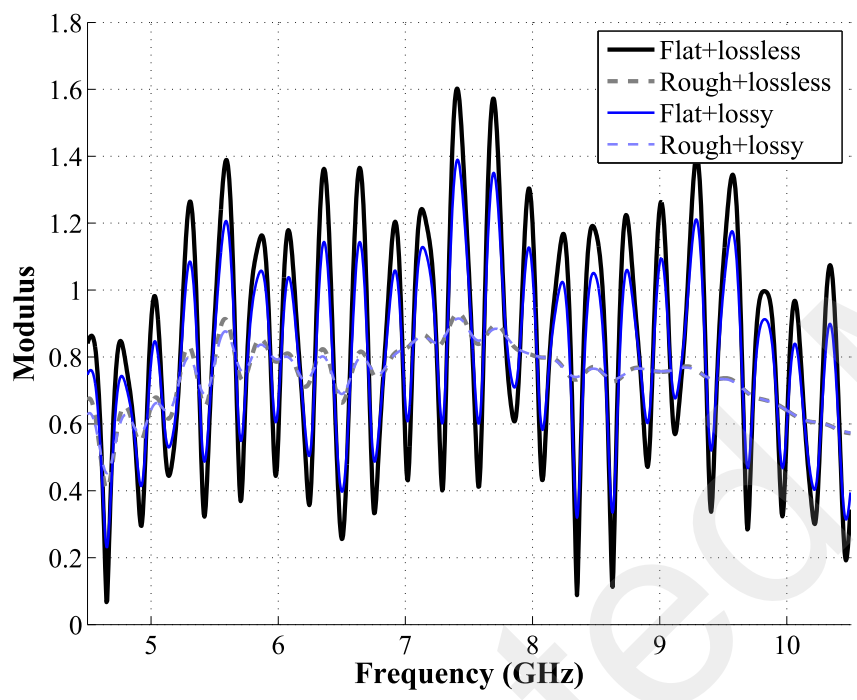

Fig. 13. Modulus (in V/m) of the GPR signal in the frequency domain for the four cases (1-4), in the frequency band $f \in[4.5 ; 10.5] \mathrm{GHz}$.

and $[0.5 ; 10.5] \mathrm{GHz}$. The used pulse is a Ricker pulse [29] with central frequency equal to the middle of the frequency band. In order to observe the time signal (usually called A-scan), an inverse Fourier transform (IFT) is carried out on the frequency responses obtained by the asymptotic EM modeling. Thus, first, simulations are presented in the frequency domain, and then in the time domain.

In the simulations, four cases will be compared, by considering either flat or rough surfaces and either lossless or lossy media (i.e., media without or with conductivity $\sigma$ ): 1) without roughness nor conductivity; 2) with roughness and without conductivity; 3) without roughness and with conductivity; and 4) with roughness and conductivity.

Fig. 12 shows the modulus (in $\mathrm{V} / \mathrm{m}$ ) of frequency response of the total coherent scattered field $\sum_{k=1}^{5}\left\langle s_{k}\right\rangle$ (called the GPR signal) for all the four cases (1-4) for the frequency band $f \in[0.5 ; 4.5] \mathrm{GHz}$. The upper part of the larger band $f \in$ $[0.5 ; 10.5] \mathrm{GHz}$ is shown in Fig. 13 for the better clarity of

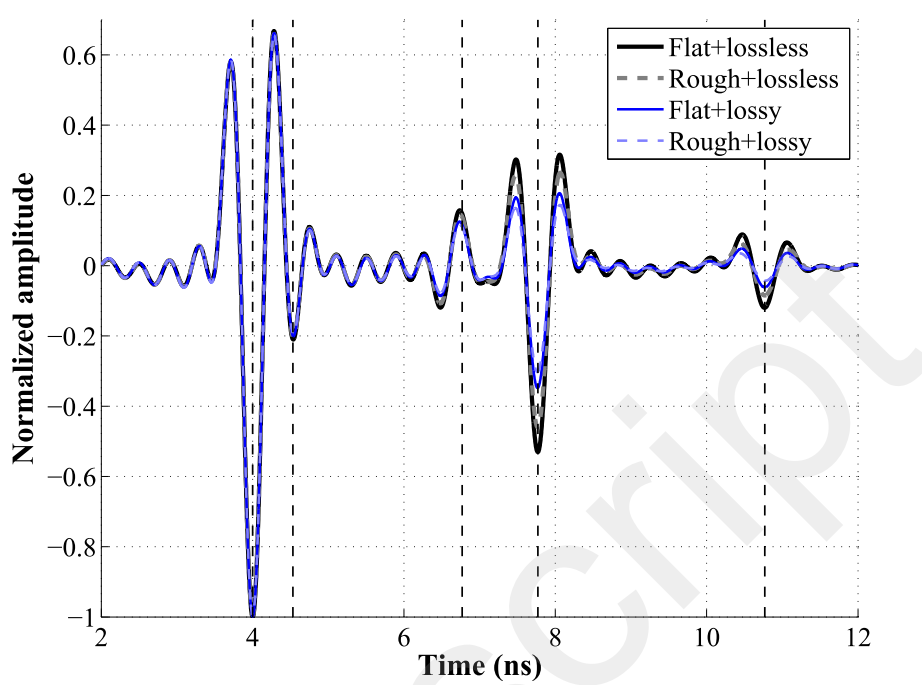

Fig. 14. Normalized GPR signal in the time domain (A-scan) for the four cases (1-4), for the frequency band $f \in[0.5 ; 2.5] \mathrm{GHz}$. Black dashed vertical lines represent the time delays in the context of flat interfaces and lossless layers.

the figure. These two figures show that when the multilayered medium is lossy, the signal modulus decreases by comparison with that for a lossless multilayered medium, for both the flat and rough surfaces. This is physically mainly due to the propagation losses inside the lossy multilayered medium, which are mathematically translated by the fact that $\epsilon_{r p}$ becomes complex in (5), so that $\Delta \phi_{k}$ also becomes complex and induces a decrease in the modulus of $s_{k}$ in (6). It can also be seen that when the multilayered medium is made up of rough interfaces, the modulus of the total coherent scattered field is generally damped compared with that of flat interfaces. Physically, this can be attributed to the fact that the roughness induces a spreading of the incident energy in directions away from the specular direction at each interface. Mathematically, it corresponds to the term accounting for the roughness $\left\langle e^{i \delta \phi_{k}}\right\rangle$, which checks the property $0<\left|\left\langle e^{i \delta \phi_{k}}\right\rangle\right|<1$. It can be seen that this damping gets stronger as the frequency increases, as predicted by the mathematical expression of $\left\langle e^{i \delta \phi_{k}}\right\rangle$. Numerical results for the higher frequencies $f \in[4.5 ; 10.5] \mathrm{GHz}$ in Fig. 13 strongly highlight this fact, as for $f>9 \mathrm{GHz}$, the oscillations are totally smoothed for both the lossless and lossy media.

Now, let us focus on time-domain numerical simulations called A-scans. Fig. 14 shows the A-scan GPR signal for the four cases and for the frequency band $f \in[0.5 ; 2.5] \mathrm{GHz}$. Normalization is applied, by dividing the data for each case by its maximum absolute value. The vertical black dashed lines represent the time delays in the context of a multilayered medium composed of flat interfaces and lossless layers. As expected, it can be seen that the first two echoes are overlapping. Indeed, for the first two echoes, the product $B \Delta \tau$ is less than 1 [30], with $B$ the frequency bandwidth ( $2 \mathrm{GHz}$ here) and $\Delta \tau$ the time shift between the first two echoes. Like in the frequency domain, when the multilayered medium is lossy, the signal amplitude decreases by comparison with that for lossless media (the same real relative permittivities $\epsilon_{r}^{\prime}$ ). 


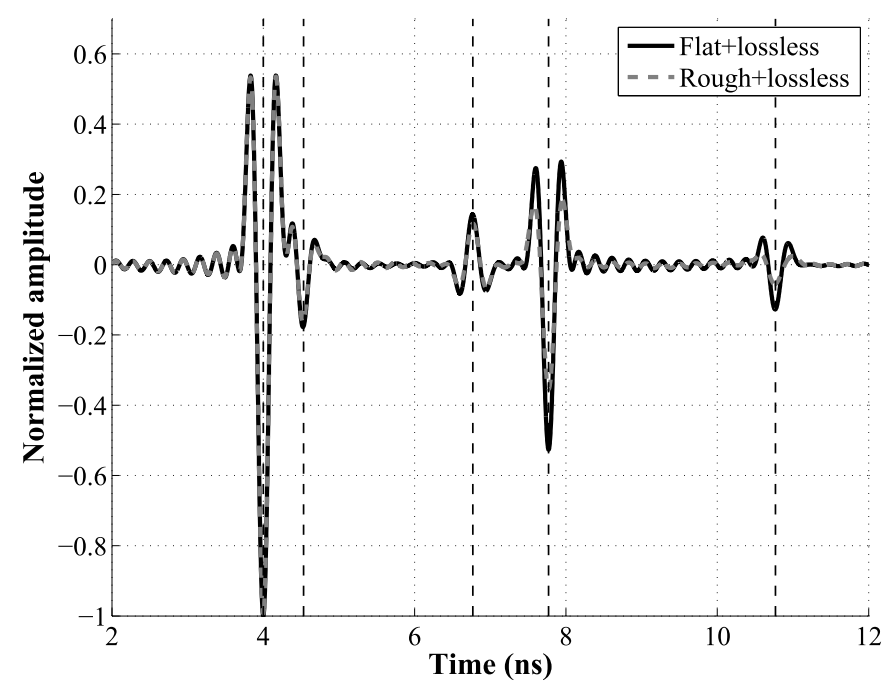

Fig. 15. Similar parameters as in Fig. 14, but the frequency band $f \in$ $[0.5 ; 4.5] \mathrm{GHz}$; only the cases without conductivity (lossless media) are plotted here.

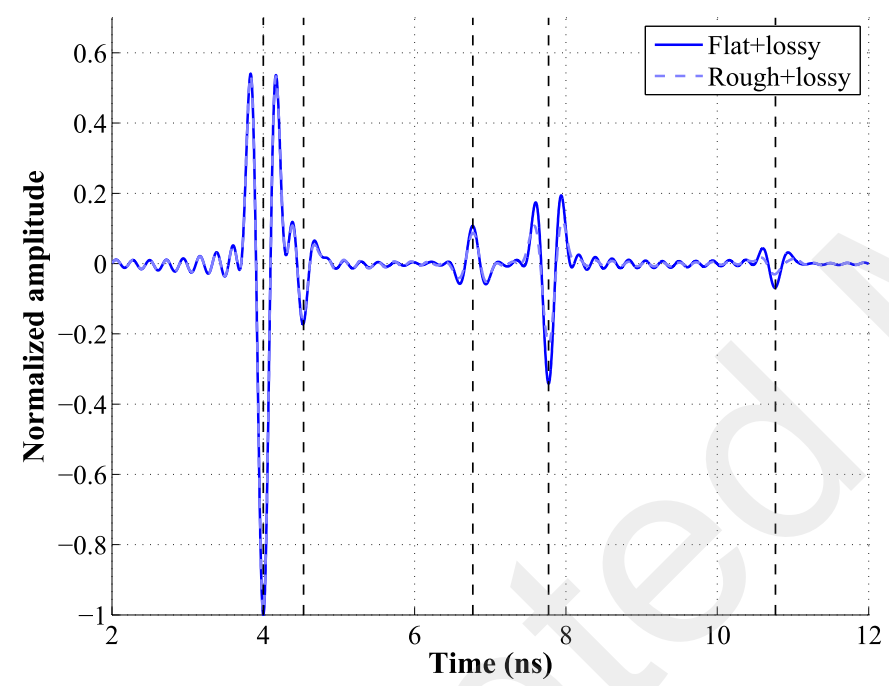

Fig. 16. Similar parameters as in Fig. 15, but for the cases with conductivity (lossy media).

Indeed, for the fourth echo, the maximum amplitude decreases to $34.4 \%$ and $34.3 \%$ for the flat and rough cases, respectively. When the multilayered medium has rough interfaces, the GPR signal is damped, and this damping gets stronger as the number of the echo increases. For example, the maximum amplitude of the fourth echo is reduced by $13.2 \%$ and $13.0 \%$ for the lossless and lossy cases, respectively. The fifth echo is more significantly reduced (by $29.3 \%$ and $30.4 \%$ for the lossless and lossy cases, respectively), because the reduction due to the interface roughness is stronger. Thus, the estimation of the propagation speed inside each layer by the method in [3] with these reductions will be biased $a$ priori.

Figs. 15 and 16 show the A-scan GPR signals with a larger frequency band: $[0.5 ; 4.5] \mathrm{GHz}$, the lossless cases being shown in Fig. 15 and the lossy cases in Fig. 16 for better clarity. For this frequency band, the first two echoes

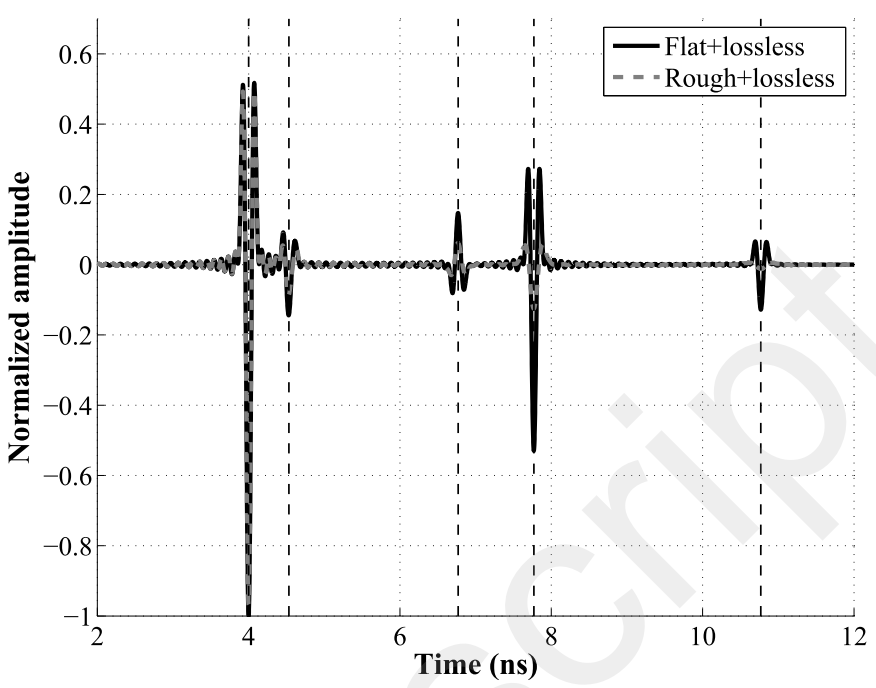

Fig. 17. Similar parameters as in Fig. 14, but the frequency band $f \in$ $[0.5 ; 10.5] \mathrm{GHz}$; only the cases without conductivity (lossless) are plotted here.

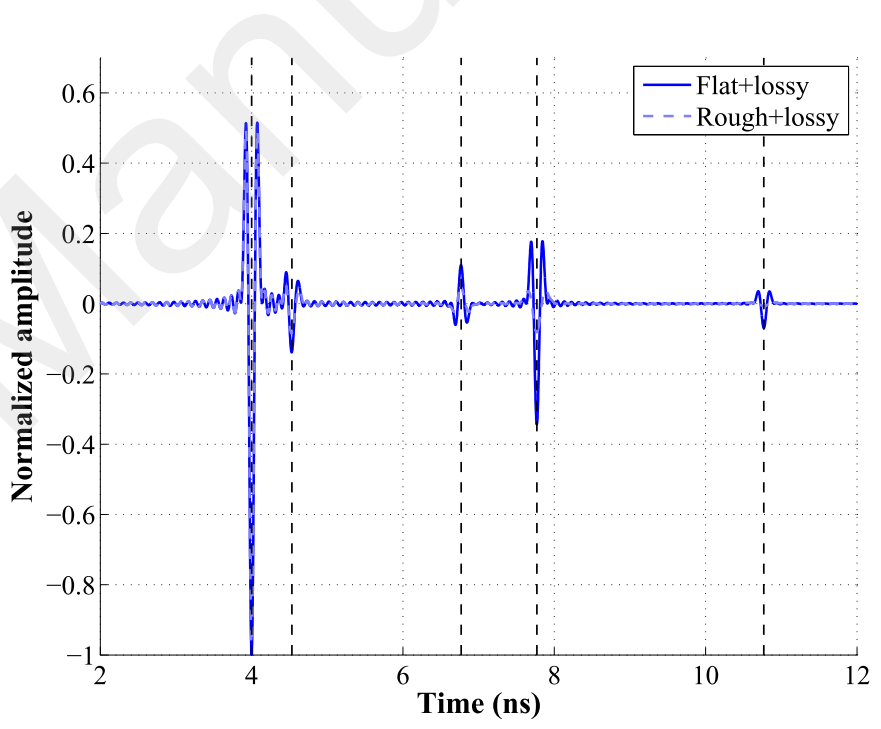

Fig. 18. Similar parameters as in Fig. 17, but for the cases with conductivity (lossy).

become nonoverlapping, and there will not be any diagnosis error anymore. In the two cases without roughness, the five interfaces can be detected by the maximum of the signal. As shown in Fig. 14, the roughness reduces the amplitude of the echoes, and the reduction becomes stronger for increasing the number of the echo (the second echo is more strongly reduced than the first one and so on), as predicted by (17) for this configuration. Then, the fifth echo becomes more hardly detectable for rough interfaces. It can be noted that the simulations have been carried out without noise; the detection of the fifth echo will be even more difficult with noise. This observation can also be made from Figs. 17 and 18, in which the frequency band is further enlarged to $[0.5 ; 10.5] \mathrm{GHz}$. First, it can be seen that the pulsewidth makes it possible to easily detect the first layer (with thickness $\bar{H}_{2}=40 \mathrm{~mm}$ ). In addition, these two figures show that the five interfaces 
are detected when the interfaces are flat. The roughness has a very significant impact here on the amplitudes of the echoes, the fifth interface being almost undetectable in this case. Thus, these simulations highlight that, in the context of a multilayered medium, the impact of the interface roughness is not necessarily negligible (in particular for large bands), and this impact gets stronger as the band gets larger.

\section{CONCLUSION}

In this article, a new asymptotic modeling of EM wave coherent scattering from a rough multilayered medium, based on the SKA, has been proposed. The very low computational burden of this method is an important advantage as compared with a rigorous numerical method. This method allows us to investigate the effects of the interface roughness on the amplitude of the echoes of the multilayered medium coming from the GPR. In particular, it has been highlighted that the roughness influence gets stronger as the frequency band is increased, and that the damping increases with the order of the echo. In this perspective, this asymptotic method can then be used with inversion methods to estimate the various medium parameters, such as the roughness of the interfaces.

\section{ACKNOWLEDGMENT}

This work may contribute to COST Action TU1208 Civil Engineering Applications of Ground Penetrating Radar.

\section{REFERENCES}

[1] I. L. Al-Qadi and S. Lahouar, "Measuring layer thicknesses with GPR-Theory to practice," Construct. Building Mater., vol. 19, no. 10, pp. 763-772, 2005

[2] C.-P. Kao, J. Li, Y. Wang, H. Xing, and C. R. Liu, "Measurement of layer thickness and permittivity using a new multilayer model from GPR data," IEEE Trans. Geosci. Remote Sens., vol. 45, no. 8, pp. 2463-2470, Aug. 2007.

[3] S. Lahouar and I. L. Al-Qadi, "Automatic detection of multiple pavement layers from GPR data," NDT \& E Int., vol. 41, no. 2, pp. 69-81, 2008.

[4] A. Benedetto and F. Benedetto, "Application field-specific synthesizing of sensing technology: Civil engineering application of groundpenetrating radar sensing technology," in Comprehensive Materials Processing, S. Hashmi, G. F. Batalha, C. J. Van Tyne, B. S. Yilbas, Eds. Amsterdam, The Netherlands: Elsevier, 2014, chs. 13.15, pp. 393-425.

[5] D. B. Oliveira, D. A. G. Vieira, A. C. Lisboa, and F. Goulart, "A well posed inverse problem for automatic pavement parameter estimation based on GPR data," NDT\&E Int., vol. 65, pp. 22-27, Jul. 2014.

[6] S. Lambot, E. Slob, M. Vanclooster, J. A. Huisman, and H. Vereecken, "Hydrogeophysical techniques for site characterization and monitoring: Recent advances in ground-penetrating radar," in Soil Chemical Pollution, Risk Assessment, Remediation and Security (NATO Science for Peace and Security Series), L. Simeonov and V. Sargsyan, Eds. Dordrecht, The Netherlands: Springer, 2008, pp. 183-202.

[7] J. Minet, P. Bogaert, M. Vanclooster, and S. Lambot, "Validation of ground penetrating radar full-waveform inversion for field scale soil moisture mapping," J. Hydrol., vols. 424-425, pp. 112-123, Mar. 2012.

[8] K. Z. Jadoon, S. Lambot, M. Dimitrov, and L. Weihermüller, "Temporal monitoring of the soil freeze-thaw cycles over snow-cover land by using off-ground GPR," in Proc. 7th Int. Workshop Adv. Ground Penetrating Radar (IWAGPR), Jul. 2013, pp. 1-4.

[9] A. Giannopoulos, "Modelling ground penetrating radar by GprMax," Construct. Building Mater, vol. 19, no. 10, pp. 755-762, Dec. 2005.

[10] N. Déchamps, N. de Beaucoudrey, C. Bourlier, and S. Toutain, "Fast numerical method for electromagnetic scattering by rough layered interfaces: Propagation-inside-layer expansion method," J. Opt. Soc. Amer. A, Opt. Image Sci., vol. 23, no. 2, pp. 69-359, Feb. 2006.
[11] C. Bourlier, C. Le Bastard, and N. Pinel, "Full wave PILE method for the electromagnetic scattering from random rough layers," in Proc. 15 th Int. Conf. Ground Penetrating Radar, Brussels, Belgium, Jun./Jul. 2014, pp. 545-551.

[12] C. Bourlier, C. L. Bastard, and V. Baltazart, "Generalization of PILE method to the EM scattering from stratified subsurface with rough interlayers: Application to the detection of debondings within pavement structure," IEEE Trans. Geosci. Remote Sens., vol. 53, no. 7, pp. 4104-4115, Jul. 2015.

[13] B. Yang and C. Rappaport, "Response of realistic soil for GPR applications with 2-D FDTD," IEEE Trans. Geosci. Remote Sens., vol. 39, no. 6, pp. 1198-1205, Jun. 2001.

[14] N. Pinel, C. Bourlier, and J. Saillard, "Forward radar propagation over oil slicks on sea surfaces using the Ament model with shadowing effect,' Prog. Electromagn. Res., vol. 76, pp. 95-126, 2007.

[15] N. Pinel, C. Bourlier, and J. Saillard, "Degree of roughness of rough layers: Extensions of the Rayleigh roughness criterion and some applications," Prog. Electromagn. Res. B, vol. 19, pp. 41-63, Jan. 2010.

[16] N. Pinel, C. Le Bastard, C. Bourlier, and M. Sun, "Asymptotic modeling of coherent scattering from random rough layers: Application to road survey by GPR at nadir," Int. J. Antennas Propag., vol. 2012, Nov. 2012, Art. no. 874840 .

[17] A. Tabatabaeenejad, X. Duan, and M. Moghaddam, "Coherent scattering of electromagnetic waves from two-layer rough surfaces within the Kirchhoff regime," IEEE Trans. Geosci. Remote Sens., vol. 51, no. 7, pp. 3943-3953, Jul. 2013.

[18] B. Sai and L. P. Ligthart, "GPR phase-based techniques for profiling rough surfaces and detecting small, low-contrast landmines under flat ground," IEEE Trans. Geosci. Remote Sens., vol. 42, no. 2, pp. 318-326, Feb. 2004.

[19] A. G. Yarovoy, C. N. Vazouras, J. G. Fikioris, and L. P. Ligthart, "Numerical simulations of the scattered field near a statistically rough air-ground interface," IEEE Trans. Antennas Propag., vol. 52, no. 3, pp. 780-789, Mar. 2004.

[20] S. Lambot, M. Antoine, M. Vanclooster, and E. C. Slob, "Effect of soil roughness on the inversion of off-ground monostatic GPR signal for noninvasive quantification of soil properties," Water Resour. Res., vol. 42, no. 3, 2006, Art. no. w03403.

[21] A. Giannopoulos and N. Diamanti, "Numerical modelling of groundpenetrating radar response from rough subsurface interfaces," Near Surf. Geophys., vol. 6, no. 6, pp. 357-369, Dec. 2008.

[22] J. van der Kruk, H. Vereecken, N. Diamanti, and A. Giannopoulos, "Influence of interface roughness and heterogeneities on the waveguide inversion of dispersive GPR data," in Proc. Int. Conf. Ground Penetrating Radar (GPR), Jun. 2010, pp. 1-5.

[23] N. Pinel, C. Le Bastard, V. Baltazart, C. Bourlier, and Y. Wang, "Influence of layer roughness for road survey by ground penetrating radar at nadir: Theoretical study," IET Radar, Sonar Navigat., vol. 5, no. 6, pp. 650-656, 2011.

[24] F. Jonard, L. Weihermüller, H. Vereecken, and S. Lambot, "Accounting for soil surface roughness in the inversion of ultrawideband off-ground GPR signal for soil moisture retrieval," Geophysics, vol. 77, no. 1, pp. H1-H7, 2012.

[25] J. van der Kruk, N. Diamanti, A. Giannopoulos, and H. Vereecken, "Inversion of dispersive GPR pulse propagation in waveguides with heterogeneities and rough and dipping interfaces," J. Appl. Geophys., vol. 81, pp. 88-96, Jun. 2012.

[26] P. Imperatore, A. Iodice, and D. Riccio, "Electromagnetic wave scattering from layered structures with an arbitrary number of rough interfaces," IEEE Trans. Geosci. Remote Sens., vol. 47, no. 4, pp. 1056-1072, Apr. 2009.

[27] N. Pinel and C. Bourlier, "Scattering from very rough layers under the geometric optics approximation: Further investigation," J. Opt. Soc. Amer. A, Opt. Image Sci., vol. 25, no. 6, pp. 1293-1306, Jun. 2008.

[28] C. Bourlier, N. Pinel, and G. Kubické, Method of Moments for 2D Scattering Problems: Basic Concepts and Applications. London, U.K.: Wiley, 2013.

[29] P.-S. Yang, S.-W. Liu, and J.-C. Sung, "Transient response of SH waves in a layered half-space with sub-surface and interface cracks," Appl. Math. Model., vol. 32, no. 4, pp. 595-609, Apr. 2008.

[30] C. Le Bastard, V. Baltazart, Y. Wang, and J. Saillard, “Thin-pavement thickness estimation using GPR with high-resolution and superresolution methods," IEEE Trans. Geosci. Remote Sens., vol. 45, no. 8, pp. 2119-2511, Aug. 2007. 


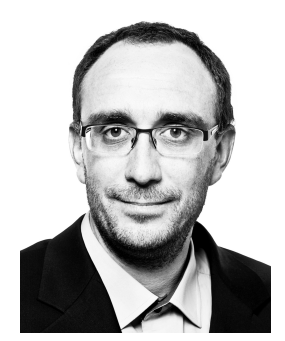

Nicolas Pinel received the Engineering degree and the M.S. degree in electronics and electrical engineering from the École polytechnique de l'université de Nantes (Polytech Nantes), Nantes, France, in 2003, and the Ph.D. degree from the University of Nantes, Nantes, in 2006.

He was with the Institut d'Électronique et de Télécommunications de Rennes (IETR) Laboratory, Nantes, for seven years, and Alyotech, Rennes, France, for four years. In 2017, he joined Icam, Carquefou, France, where he works as an Associate Professor. His research interests are in the areas of radar and optical remote sensing, scattering and propagation. In particular, he works on asymptotic methods of electromagnetic wave scattering from random rough surfaces and layers.

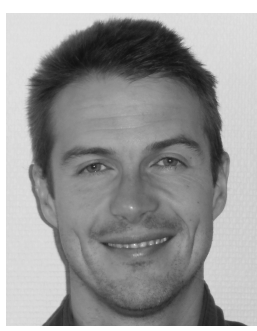

Cédric Le Bastard received the B.S. and M.S. degrees in electronic engineering from the University of Rennes, Rennes, France, in 2001 and 2003, respectively, and the Ph.D. degree from the Laboratoire Central des Ponts et Chaussées, University of Nantes, Nantes, France, in 2007.

$\mathrm{He}$ is currently with the Centre for Expertise and Engineering on Risks, Environment, Mobility, Urban and Country Planning (Cerema), Les Ponts de Cé, France. His research interests include radar, nondestructive testing and evaluation, electromagnetic wave scattering, time delay estimation, and array signal processing.

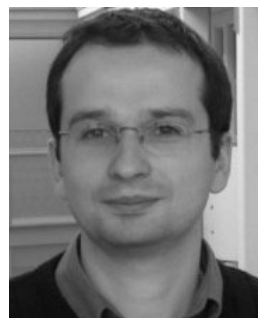

Christophe Bourlier was born in La Flèche, France, in July 6, 1971. He received the M.S. degree in electronics from the University of Rennes, Rennes, France, in 1995, and the Ph.D. degree from the Systèmes Electroniques et Informatique (SEI) Laboratory, Nantes, France, in 1999.

$\mathrm{He}$ is currently with the Institute of Electronics and Telecommunications of Rennes (IETR) Laboratory, Polytech Nantes, University of Nantes, Nantes. $\mathrm{He}$ is also a Researcher with the National Center for Scientific Research, working on electromagnetic wave scattering from rough surfaces (ocean-like surfaces) and objects for microwaves and infrared remote sensing applications and radar signatures. He has authored more than 180 journal and conference articles. 